Article

\title{
An Optimization of Two-Dimensional Photonic Crystals at Low Refractive Index Material
}

\author{
Thanh-Phuong Nguyen ${ }^{1}$, Tran Quoc Tien ${ }^{2}$ and Quang Cong Tong ${ }^{2, *}$ and Ngoc Diep Lai ${ }^{3, *}$ \\ 1 School of Engineering Physics, Hanoi University of Science and Technology, No. 1 Dai Co Viet, \\ Hai Ba Trung, Hanoi 100000, Vietnam \\ 2 Institute of Materials Science, Vietnam Academy of Science and Technology, 18 Hoang Quoc Viet, \\ Cau Giay, Hanoi 100000, Vietnam \\ 3 Laboratoire de Photonique Quantique et Moléculaire, UMR 8537, École Normale Supérieure Paris-Saclay, \\ Centrale Supélec, CNRS, Université Paris-Saclay, 61 avenue de Président Wilson, 94235 Cachan, France \\ * Correspondence: congtq2004@gmail.com (Q.C.T.); ngoc-diep.lai@ens-paris-saclay.fr (N.D.L.)
}

Received: 1 August 2019; Accepted: 21 August 2019; Published: 24 August 2019

\begin{abstract}
Photonic crystal (PC) is usually realized in materials with high refractive indices contrast to achieve a photonic bandgap (PBG). In this work, we demonstrated an optimization of two-dimensional PCs using a low refractive index polymer material. An original idea of assembly of polymeric multiple rings in a hexagonal configuration allowed us to obtain a circular-like structure with higher symmetry, resulting in a larger PBG at a low refractive index of 1.6. The optical properties of such newly proposed structure are numerically calculated by using finite-difference time-domain (FDTD) method. The proposed structures were realized experimentally by using a direct laser writing technique based on low one-photon absorption method.
\end{abstract}

Keywords: photonic crystals; photonic bandgaps; polymer materials; direct laser writing

\section{Introduction}

Photonic crystal (PC), an artificial material in which the refractive index is modulated at wavelength scale, offers presently many interesting applications in different domains $[1,2]$. The most important property of the PCs is the existence of a so-called photonic bandgap (PBG). In case of one-dimensional (1D) PCs, the PBG can be easily obtained by assembling multiple layers of two different materials with very low refractive indices contrast [3-6]. However, in case of two- and three-dimensional (2D and 3D) PCs, it requires that the refractive indices contrast should be as high as possible. Some 2D and 3D PCs are made by polystyrenes or $\mathrm{SiO}_{2}$ nanoparticles [7-10], which are fabricated by a simple fabrication method, but these PCs do not have a true PBG, or in other word, they have a so-called pseudo or partial PBG, due to their weak refractive index. Therefore, most 2D and 3D PCs are made by semiconductor materials with high refractive indices, which however makes the fabrication procedure complicated and expensive. Different optimizations have been proposed to obtain the PBG as large as possible and with a material with a smaller refractive index. By increasing the symmetry of the PCs, for example in the case of 2D PCs, from square (four-fold) to hexagon (six-fold), and to quasi-periodic PCs, such as Penrose structure [11,12], the PBG can be opened at lower refractive index materials, such as polymers $[13,14]$.

The most difficult task of PCs investigations is the fabrication, which should satisfy several conditions, such as simple, rapid, inexpensive, and particularly flexible. Today, many fabrication techniques have been proposed to realize PCs, for example, e-beam lithography, self-assembly of opals, laser interference, and direct laser writing. The choice of fabrication technique depends on desired applications. Among available fabrication technologies, the direct laser writing (DLW) is the most 
powerful one allowing fabrication of any desired PCs, in multi-dimension ((1D, 2D, 3D) PCs) [15,16]. The working principle of the DLW is to focus tightly a laser beam into a small region of a photoresist to induce a local polymerization or depolymerization effect. By moving the focusing spot following a well-designed structure, a corresponding polymeric pattern can be obtained.

In this work, we demonstrated an optimization of the 2D PCs to obtain a large PBG even with low refractive index materials, such as polymer. We proposed an original idea of high symmetry 2D PCs, and a new experimental way to realize these PCs by the DLW technique. The PBGs of a standard honeycomb structure and of a newly proposed structure are calculated and compared by using a Finite-difference time-domain (FDTD) method. These structures are then fabricated by a new scanning method using the one-photon absorption-based DLW technique [17].

\section{Theoretical Calculation}

For calculation of the PBG of different PCs, we have used the FDTD method (Lumerical Software) with Bloch boundary conditions. We assumed that the refractive index of material is 1.6, a typical value of polymer without considering the dispersion effect of the material. This choice is justified by the values experimentally measured in our case, i.e.; the refractive index of the used polymer is in between 1.55 and 1.62 for the wavelengths ranging from $400 \mathrm{~nm}$ to $800 \mathrm{~nm}$. For simulations of 2D PCs, we distinguished two different modes, TM and TE, which are the transverse magnetic or transverse electric modes where the magnetic or electric field is parallel to the PC plane, respectively.

It is well-known that the honeycomb lattice is the best 2D PC, which provides a largest PBG, as compared to the square or hexagonal lattice. We first calculated the PBG of this structure at low refractive index material, by considering two types: one is the honeycomb lattice comprising of polymeric cylinders $(n=1.6)$ in an air background $(n=1)$ and the another is an inverse geometry consisting of air-holes in the polymeric background. Figure 1a,b show a honeycomb lattice and its reciprocal space, where $\mathrm{a}$ is lattice constant, and $\mathrm{r}$ is the radius of polymeric cylinders or air-holes. For the case of polymeric cylinders in the air background, the PBG only reveals a small gap for TE mode. Figure 2a shows a band structure for TE mode, obtained with an optimum radius of $r / a=0.3$. Figure $2 b$ shows gap map obtained with various filling factors (different ratios of $\mathrm{r} / \mathrm{a}$ ). We see that the largest PBG for TE mode is about 0.02 at $\mathrm{r}=0.3 \mathrm{a}$. When considering a honeycomb lattice made of air cylinders in the polymeric background, we found that the PBG only reveals for TM mode. A band structure for TM mode $(r / a=0.35)$ is shown in Figure 3a. The PBG (for TM mode) as a function of the r/a ratio was also calculated, and a gap map is presented in the Figure $3 b$. We can see that the PBG is increased as compared to the one obtained in previous case, and reaches 0.05 at $r=0.35$ a. These results are somehow similar to results obtained with higher refractive index [1,2]. But at low refractive index material, the PBG exists but not complete, i.e.; no common PBG for both TE and TM modes. Table 1 summarizes the PBG of the honeycomb lattice at low refractive index material for both configurations.

(a)

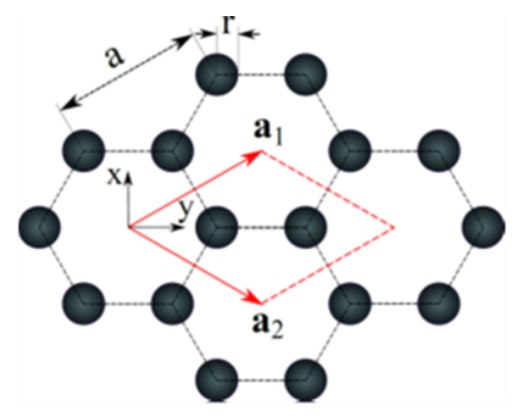

(b)

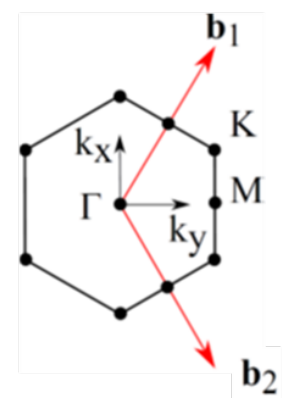

Figure 1. (a) A two-dimensional honeycomb lattice with two main lattice vectors: $\mathbf{a}_{1}=[\sqrt{3} a / 2, a / 2]$, $\mathbf{a}_{2}=[\sqrt{3} \mathrm{a} / 2,-\mathrm{a} / 2]$. The artificial atome has a cylindrical form with a radius $\mathrm{r}$. (b) The reciprocal space of corresponding two-dimensional honeycomb lattice with the primitive vectors: $\mathbf{b}_{1}=2 \pi / \mathrm{a}(1 / 3 \sqrt{3}$, $1 / 3)$ and $\left.\mathbf{b}_{2}=2 \pi / \mathrm{a}(1 / 3 \sqrt{3},-1) / 3\right)$. The reduced first Brillouin zone is identified by $\Gamma, \mathrm{K}$ and $\mathrm{M}$ points. 
(a)

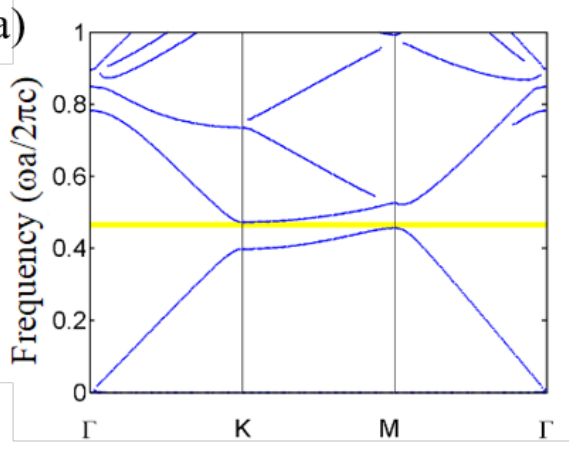

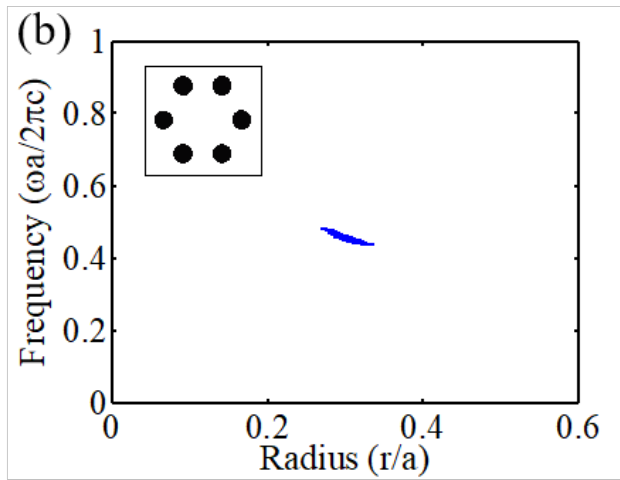

Figure 2. Photonic band structureof a $2 \mathrm{D}$ honeycomb lattice of polymeric cylinders in an air background (see insert of b). (a) Photonic bandgap for TE mode with $\mathrm{r} / \mathrm{a}=0.3$. (b) Map of photonic bandgap as a function of $\mathrm{r} / \mathrm{a}$ (shaded blue region).

(a)

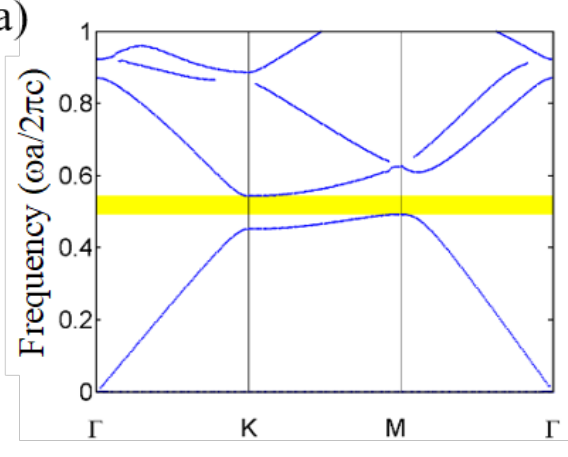

(b)

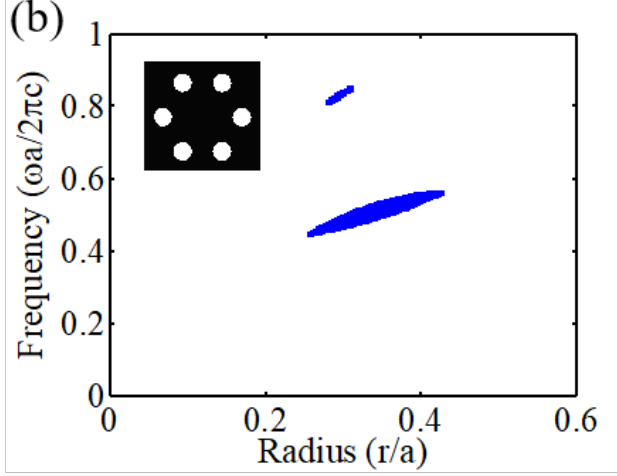

Figure 3. Photonic band structure of a 2D honeycomb lattice of air cylinders in a polymeric background (see insert of b). (a) Photonic bandgap for TM mode with $\mathrm{r} / \mathrm{a}=0.35$. (b) Map of photonic bandgap as a function of $\mathrm{r} / \mathrm{a}$ (shaded blue region).

Table 1. Summary of the best photonic bandgaps of honeycomb lattices obtained with a polymer material with a refractive index of $n=1.6$.

\begin{tabular}{cccc}
\hline Polarization Mode & Type of Cylinders & The Existence of PBG & The Largest PBG \\
\hline TM & Air & Yes & 0.05 \\
TM & Polymer & No & NA \\
TE & Air & No & NA \\
TE & Polymer & Yes & 0.01 \\
\hline
\end{tabular}

It has been well-known that the larger PBG can be obtained by increasing the structure symmetry [18]. Different structures have been proposed [11-14], and larger PBG are obtained, even at low refractive index materials. In particular, it is important to emphasis on the most symmetric one, called circular photonic crystal (CPC) [19,20], which possesses a perfect symmetry in a 2D plane. This CPC structure enables particularly an isotropic PBG, and consequently a PBG at low refractive index. However, the CPC is a non-periodic structure and usually developed around a symmetric point. Therefore, its photonic property is only characterized by a transmission calculation or measurement but not its PBG.

In this work, we proposed a novel kind of equivalent 2D PC by arranging multi-rings in a periodic configuration, such as a hexagonal lattice. This novel structure allowed us to combine the advantages of both CPC structure, thanks to the perfect symmetry of the rings (dielectric or air), and the periodic organization of these rings in a well-known hexagonal lattice, which can be numerically simulated to 
obtain a true PBG. As it will be shown in the experimental part, the proposed structure is also matched to the capacity of the fabrication technology.

Figure $4 \mathrm{a}$ presents an assembled multi-rings lattice and Figure $4 \mathrm{~b}$ illustrates its corresponding reciprocal space, where a is the lattice constant, $\mathrm{r}$ is the radius of the rings, and $\mathrm{d}$ is the width of rings. Similar to honeycomb PC, the PBG of the assembled multi-rings lattice can be calculated for two possible configurations: the lattice containing multi-rings of air $(n=1)$ in the polymeric background $(n=1.6)$ and the another is an inverse geometry consisting of multi-rings of polymer in the air background. In practices, these two configurations can be optically realized by using a negative photoresist or a positive photoresist, or simply by using a positive photoresist with controllable laser power [17]. We demonstrated that the multi-rings assembly allowed obtaining different 2D PCs with various filling factors. That can be done by changing the $r / a$ ratio. It is clear that if a $>2 r$, all rings will be separated, and the structure is a simple hexagonal PC with a ring as an "artificial atom". However, if a $<2 r$, multiple rings will overlap to each other, resulting in a complicated 2D PC, with a perfect symmetry determined by the ring.

(a)

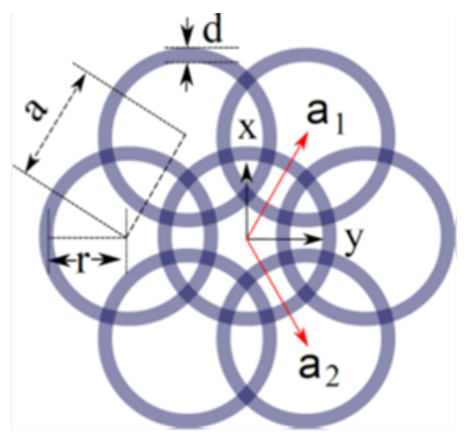

(b)

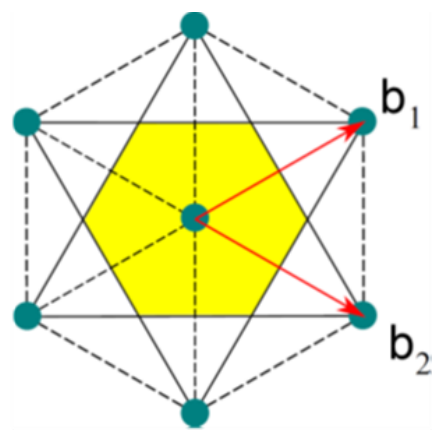

Figure 4. (a) Proposal of a 2D assembled-multirings structure arranged in a hexagonal configuration. The main lattice vectors are $\mathbf{a}_{1}=\mathrm{a}(1 / 2, \sqrt{3} / 2)$ and $\mathbf{a}_{2}=\mathrm{a}(1 / 2,-\sqrt{3} / 2) ; \mathrm{r}$ is the radius of rings; $\mathrm{d}$ is the width of ring-walls; $a$ is the lattice constant. (b) The corresponding $2 \mathrm{D}$ reciprocal space off the 2D assembled-multirings structure. $\mathbf{b}_{1}=2 \pi / \mathrm{a}(1,1 / \sqrt{3})$ and $\mathbf{b}_{2}=2 \pi / \mathrm{a}(1,-1 / \sqrt{3}):$ the primitive vectors of the reciprocal lattice. The Brillouin zone is identified by a yellow hexagon.

Figure 5 shows the simulation results for the case of assembled polymeric multi-rings $(n=1.6)$ in the air background. Similar to the case of the honeycomb, it has seen that the PBG reveals only for TE mode. For the simulation shown in Figure $5 \mathrm{a}$, we assumed that the ring width is $300 \mathrm{~nm}$, the ring separation $\mathrm{a}=1.5 \mu \mathrm{m}$, and the ring radius $\mathrm{r} / \mathrm{a}=0.51$, which is suitable to the fabrication capacity. A larger PBG is obtained, as compared to similar case of the honeycomb lattice shown in Figure 2a. By changing the radius of rings, a gap map (shaded blue region) as a function of $\mathrm{r} / \mathrm{a}$ for TE mode has been carried out, and shown in Figure 5b. It can be seen that the largest PBG is obtained with a value of $\mathrm{a} \Delta \omega / 2 \pi \mathrm{c}=0.035$ with $\mathrm{r}=0.51 \mathrm{a}$.

For the case of a 2D lattice consisting of air rings $(n=1)$ in the polymeric background, in contrast, the PBG reveals only for TM mode. Figure 6a shows its PBG obtained with a radius ratio of $\mathrm{r} / \mathrm{a}=0.78$ with $\mathrm{a}=1.5 \mu \mathrm{m}$. Again, the PBG is larger, as compared to that obtained by the honeycomb lattice shown in Figure 3a. A map of PBGs for TM mode versus the ratio of $r / a$ is shown in Figure $6 \mathrm{~b}$. It can be seen that multiple gaps can be obtained with this structure, even with low refractive index contrast. The PBG can reach a value of $a \omega \omega / 2 \pi \mathrm{c}=0.089$ at $\mathrm{r}=0.78 \mathrm{a}$, which is larger than 0.05 , obtained by the honeycomb PC. A summary of PBGs of multi-rings lattice is shown in Table 2. This novel PC still does not produce a complete PBG, but the individual PBG (for TE or TM mode) is better than those obtained by a best standard 2D PC (e.g., honeycomb). 
(a)

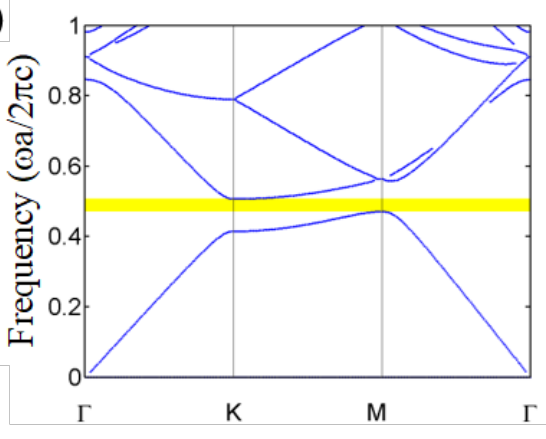

(b)

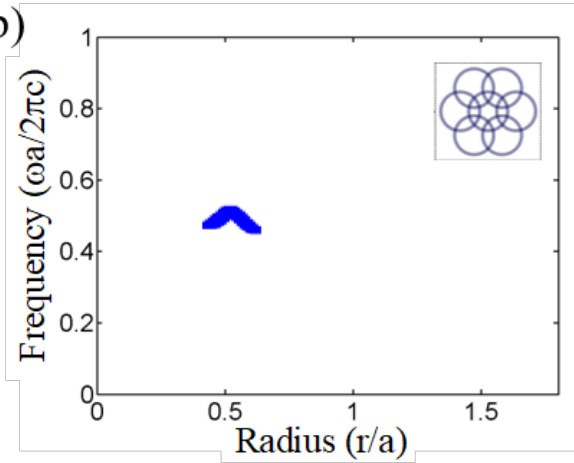

Figure 5. (a) Photonic band structure (TE mode) of an assembled-multirings lattice consisted of polymer-rings in an air background. (b) Map of photonic bandgap (TE mode) as a function of $\mathrm{r} / \mathrm{a}$ (shaded blue region). The simulations are realized with $\mathrm{r} / \mathrm{a}=0.51, \mathrm{a}=1.5 \mu \mathrm{m}, \mathrm{d}=300 \mathrm{~nm}$, and $n=1.6$.

(a)

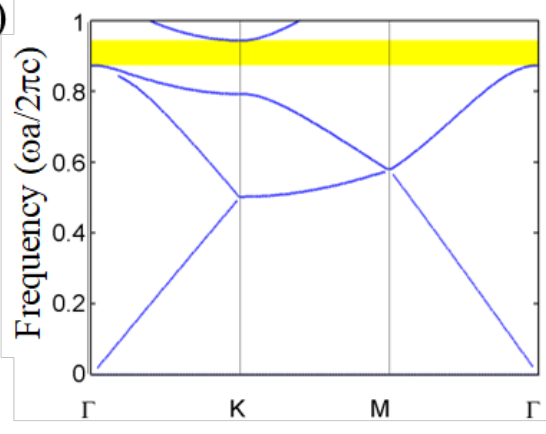

(b)

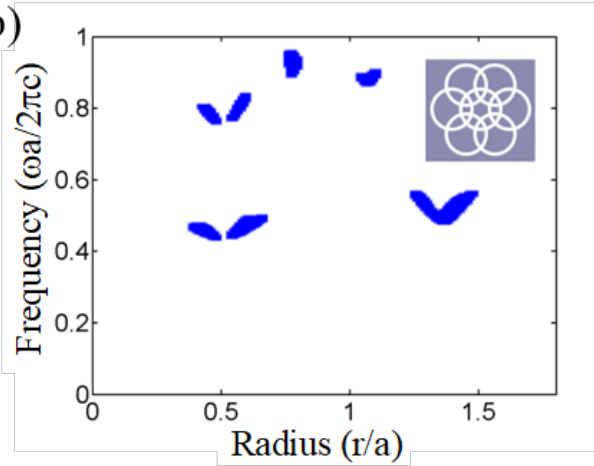

Figure 6. (a) Photonic band structure (TM mode) of an assembled-multirings lattice consisted of air rings in the polymer background. (b) Map of photonic bandgap (TM mode) as a function of $\mathrm{r} / \mathrm{a}$ (shaded blue region). The simulations are realized with $\mathrm{r} / \mathrm{a}=0.78, \mathrm{a}=1.5 \mu \mathrm{m}, \mathrm{d}=300 \mathrm{~nm}$, and $n=1.6$.

Table 2. Summary of photonic bandgaps of assembled multi-rings structures. The calculations were realized with a ring width of $\mathrm{d}=300 \mathrm{~nm}$ and the polymer material has a refractive index of $n=1.6$.

\begin{tabular}{cccc}
\hline Polarization Mode & Assembled Multi-Rings of & The Existence of PBG & The Largest PBG \\
\hline TM & Air & Yes & 0.089 \\
TM & Polymer & No & NA \\
TE & Air & No & NA \\
TE & Polymer & Yes & 0.035 \\
\hline
\end{tabular}

We note that in a standard case of square or hexagonal 2D PCs, the rod-type has a TM-bandgap and the hole-type has a TE-bandgap, respectively [1,2]. However, when working with particular structures, such as honeycomb or multi-ring PCs, the situation changes. In these cases, the existence of TM or TE bandgaps varies as a function of the ratio between the rod size and the lattice periodicity. With low refractive index, we can see that the rod-type favors a TE-bandgap while the hole-type favors a TM-bandgap, respectively.

\section{Fabrication of Proposed Structure by Direct Laser Writing Method}

To fabricate these proposed PCs, two possible methods can be used, such as e-beam lithography and DLW method. In this work, we have demonstrated the fabrication by using one-photon absorption-based DLW method, which is simple, low cost, and it can even produce 3D PCs [16,17,21]. The experimental setup is shown in Figure 7 and can be explained as follows. A simple and low-cost continuous-wave (cw) $532 \mathrm{~nm}$ laser was used to write the desired structures. The laser power was adjusted by using a combination of a half-wave plate and a polarizing beam splitter. The laser beam is 
sent through an electronic shutter (S), which allows controlling the exposure time. The quarter-wave plate was used to obtain a circular polarization of the excitation beam, just before focusing. The laser beam was sent on a 50/50 beam-splitting cube (BS), after which one part goes to a powermeter and the other is directed to an oil-immersion objective lens (OL) with a numerical aperture (NA) of 1.3, which focuses the laser beam on the photoresist sample. The last one was placed on a piezoelectric translation stage (PZT), which allows moving the sample in 3D with a resolution of $0.1 \mathrm{~nm}$. Thanks to the high NA OL, the laser is tightly focused onto the sample with a focusing spot of about $300 \mathrm{~nm}$. To determine the focusing position, the same OL is used to collect the fluorescence photons, emitted from the photoresist sample, which then propagate in opposite direction with respect to the excitation beam. The fluorescence beam is passed through a long-pass filter $(\mathrm{F})$ with a cut-off wavelength of $580 \mathrm{~nm}$, to remove the excitation laser light, and directed towards an avalanche photodiode (APD). It has been demonstrated that this technique can fabricate structures with a thickness as large as 100 micrometers, depending on the absorption coefficient of the photoresist [16,21], with a periodicity down to $400 \mathrm{~nm}$ and the smallest size of pattern can be as small as $57 \mathrm{~nm}$ [17].

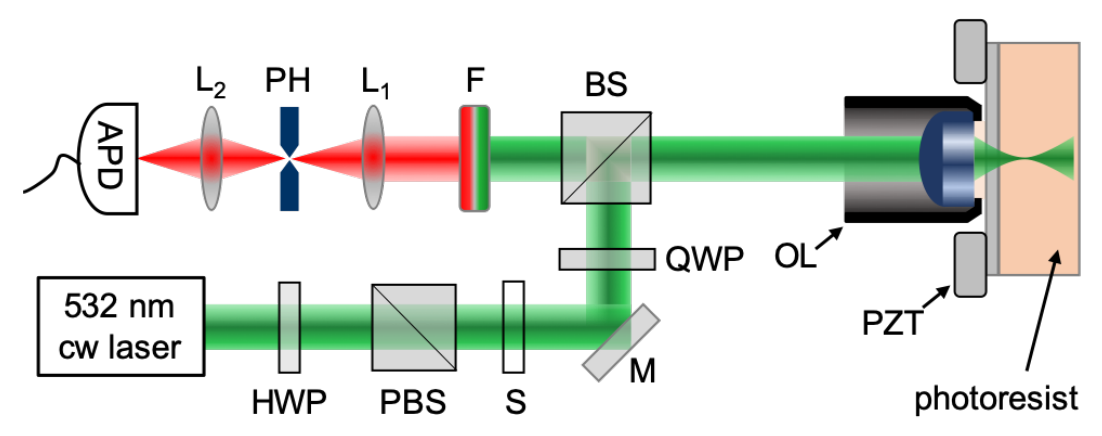

Figure 7. Experimental setup of the low one-photon absorption-based direct laser writing method used to fabricate desired polymeric photonic crystals. HWP: half-wave plate, PBS: polarizing beam splitter, S: electronic shutter, M: mirror, QWP: quarter-wave plate, BS: beam splitter, OL: objective lens, PZT: piezoelectric translator, F: long-pass filter, $\mathrm{L}_{1,2}$ : lenses, $\mathrm{PH}$ : pinhole, APD: avalanche photodiode.

In this work, we proposed a new way to realize desired structures. Indeed, in e-beam lithography or DLW, the structures are usually created by moving the focusing spot following a design. We proposed to assembly multiple lines or rings to achieve desired structures. Various structures can be created with the same lines or rings assembly, which simplifies a lot for fabrication procedure. For demonstration, we used a positive S1818 photoresist with a thickness of $1 \mu \mathrm{m}$. With low excitation laser power, the focusing spot produced an air-hole in polymer thin film (after development), and by moving this focusing, we obtained an assembly of air multi-rings lattice. Please note that with the same photoresist and with higher excitation laser power, we can obtain a polymeric multi-ring thanks to the optically induced thermal effect [17].

Figure 8a shows a new proposed trajectory of the focusing laser beam to draw a honeycomb lattice. By this method, we can obtain different structures, starting from a standard air-hole hexagonal structure to a honeycomb structure with controllable filling factors and dots shape. Figure $8 \mathrm{~b}-\mathrm{e}$ show scanning electron microscope (SEM) images of different structures (period of $a=1.5 \mu \mathrm{m}$ ) fabricated by a constant laser power of $10 \mu \mathrm{W}$ and by different moving velocities from $9 \mu \mathrm{m} / \mathrm{s}$ to $6 \mu \mathrm{m} / \mathrm{s}$. We can see that with velocities of $9 \mu \mathrm{m} / \mathrm{s}$ and $8 \mu \mathrm{m} / \mathrm{s}$, typical hexagons with air-holes are obtained, while with velocities of $7 \mu \mathrm{m} / \mathrm{s}$ and $6 \mu \mathrm{m} / \mathrm{s}$ (higher dose), honeycombs with different filling factors are demonstrated. Thus, by using the simple scanning scheme shown in Figure 8a, and by controlling the scanning speed, various types of honeycomb lattices can be obtained. 
(a)

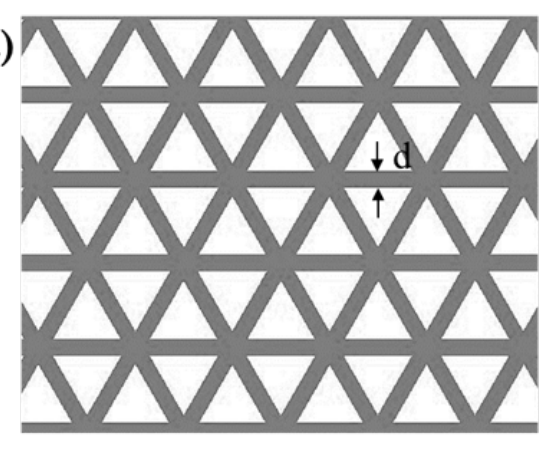

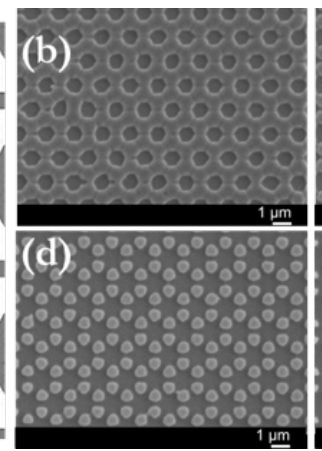

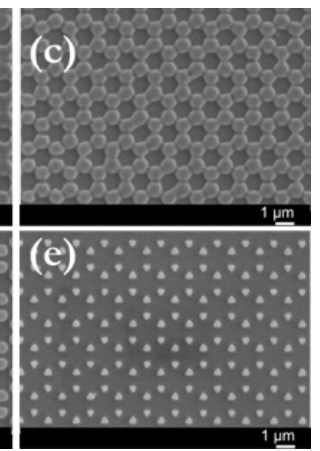

Figure 8. Fabrication of a honeycomb lattice by a simple direct laser writing method. (a) Illustration of a writing model in which the focused laser beam is scanned line by line with a period a, following a triangular configuration. The line width, $d$, is controlled by the writing parameters, such as laser power, writing speed, and focusing lens. The final structures vary from air-holes hexagonal structure to polymeric-dots honeycomb, depending on the writing doses. (b-e) SEM images of fabricated 2D structures (period, $\mathrm{a}=1.5 \mu \mathrm{m}$ ), obtained with different doses. For these structures, the laser power was fixed at $10 \mu \mathrm{W}$ and the scanning speeds were $9 \mu \mathrm{m} / \mathrm{s}(\mathbf{b}), 8 \mu \mathrm{m} / \mathrm{s}(\mathbf{c}), 7 \mu \mathrm{m} / \mathrm{s}(\mathrm{d})$, and $6 \mu \mathrm{m} / \mathrm{s}(\mathbf{e})$, respectively.

By the same method, assembled multi-rings lattices were fabricated by scanning the focusing laser beam following the trajectory shown in Figure 9a. Different structures have been demonstrated by changing the structures parameters, such as the ring width, $d$, the lattice constant, a, as well as the ring radius, r. Figure $9 \mathrm{~b}-\mathrm{e}$ show SEM images of four structures realized by keeping the same laser power, $10 \mu \mathrm{W}$ while changing the moving velocities of laser beam from $9 \mu \mathrm{m} / \mathrm{s}$ to $6 \mu \mathrm{m} / \mathrm{s}$. The lattice constant and the ring radius were chosen as $2 \mu \mathrm{m}$ and $1.51 \mu \mathrm{m}$, respectively. When varying the scanning velocities, the ring width changes from $150 \mathrm{~nm}$ (Figure 9b), $200 \mathrm{~nm}$ (Figure 9c), $300 \mathrm{~nm}$ (Figure 9d), to $400 \mathrm{~nm}$ (Figure 9e), resulting in different 2D structures with different filling factors and structure compositions. This perfectly agrees with the theoretical prediction, and their PBGs are also demonstrated to be better than those obtained by the standard 2D PCs.

(a)

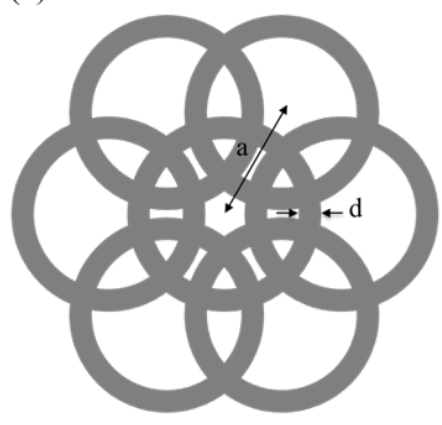

(b)

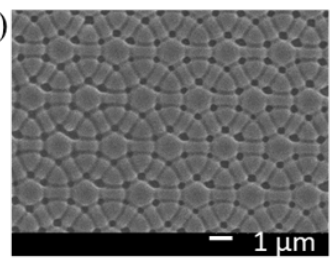

(d)

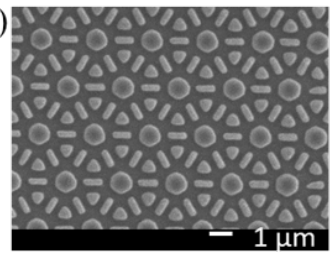

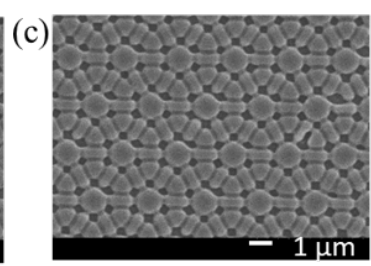

(e)

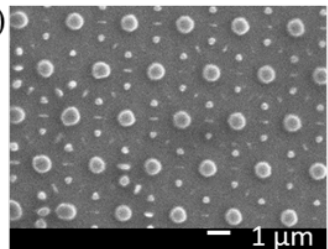

Figure 9. Fabrication of various high symmetry 2D photonic crystals by assembling multiple rings. (a) Illustration of the assembly of multi-rings, created by moving the focused laser beam, following a hexagonal configuration. The ring width, $\mathrm{d}$, is controlled by the writing dose, and the rings are separated, from center to center, by a lattice constant a. By changing the exposure doses (laser power and writing speed) as well as the lattice constant, a, the final structures can be varied from a Penrose-like structure (b) to a hexagonal structure (e) consisting of different polymeric dots. (b-e) SEM images of 2D assembled-multi-rings lattices with a lattice constant $\mathrm{a}=2 \mu \mathrm{m}$ and fabricated by the same laser power of $10 \mu \mathrm{W}$. Different structures were obtained, as predicted by the simulations, with different ring widths $\mathrm{d}=150 \mathrm{~nm}(\mathbf{b}), 200 \mathrm{~nm}(\mathbf{c}), 300 \mathrm{~nm}(\mathrm{~d})$, and $400 \mathrm{~nm}(\mathbf{e})$, realized by different scanning speeds, $9 \mu \mathrm{m} / \mathrm{s}(\mathbf{b}), 8 \mu \mathrm{m} / \mathrm{s}(\mathbf{c}), 7 \mu \mathrm{m} / \mathrm{s}(\mathbf{d})$, and $6 \mu \mathrm{m} / \mathrm{s}(\mathbf{e})$, respectively. 
Please note that it is not possible at this moment to experimentally characterize the PBGs of these fabricated structures, because the structures sizes are limited to $100 \mu \mathrm{m} \times 100 \mu \mathrm{m}$ due to the scanning system of the DLW technique. Also, with a lattice constant of $2 \mu \mathrm{m}$, an assembled multi-rings PC produces a PBG in the range of 2.85-3.15 $\mu \mathrm{m}$, which is in the mid-infrared domain. The limited PC thickness $(1 \mu \mathrm{m})$ also makes the direct PBG characterization difficult. Further reduction of the lattice constant (a) and of the ring radius (r), and increase of the PCs thickness are therefore necessary to obtain a PBG in visible or near IR range, which can be very promising for many applications. A direct application of such fabricated structures is to make PC-based organic laser, with controllable emission wavelength and desired beam shape.

\section{Conclusions}

In conclusion, we have demonstrated an optimized two-dimensional photonic crystal with a better photonic bandgap at low refractive index contrast. The numerical calculations of the photonic bandgaps of honeycombs and assembled multi-rings lattices at low refractive index $(n=1.6)$ have been done by using FDTD method. The results showed that the TM mode has larger PBGs, as compared to the TE mode, and the largest PBG is approximately $0.089(=\mathrm{a} \Delta \omega / 2 \pi \mathrm{c})$ at $\mathrm{r}=0.78 \mathrm{a}$, obtained by the newly proposed assembled multi-rings photonic crystal. This is due to the perfect symmetry at local area of the photonic crystal, and the PBG calculation is possible thanks to the organization of multiple rings in a periodic structure. Both types of lattices, honeycomb and assembled multi-rings, have then been fabricated by using a low one-photon absorption-based direct laser writing technique, in a simple way. By controlling the exposure doses (laser power and exposure time), the lattice parameters, such as structure filling factor and lattice periodicity, were well controlled, allowing an optimization of the photonic bandgap.

Author Contributions: Q.C.T., T.Q.T. and N.D.L. proposed the idea and designed the experiments. T.-P.N. performed simulations and Q.C.T. realized experiments and analyzed the data. All authors wrote, reviewed and approved the final version of manuscript.

Funding: This research is supported by a public grant of Ministry of Science and Technology of Vietnam under the project [ĐTĐLCN.01/2017] and by a public grant overseen by the French National Research Agency in the frame of GRATEOM project [ANR-17-CE09-0047-01].

Conflicts of Interest: The authors declare no conflict of interest.

\section{References}

1. Joannopoulos, J.D.; Meade R.D.; Winn, J.N. Photonic Crystals: Molding the Flow of Light; Princeton University Press: Princeton, NJ, USA, 1995.

2. Akoda, K. Optical Properties of Photonic Crystals, 2nd ed.; Springer Science \& Business Media: Berlin, Germany, 2004; ISBN 978-3-662-14324-7.

3. Gao, S.; Tang, X.; Langner, S.; Osvet, A.; Harrei, C.; Barr, M.K.S.; Spiecker, E.; Bachmann, J.; Brabec, C.J. Forberich, K. Time-resolved analysis of dielectric mirrors for vapor sensing. ACS Appl. Mater. Interfaces 2018, 10, 36398-36406 [CrossRef] [PubMed]

4. Lova, P. Selective polymer distributed bragg reflector vapor sensors. Polymers 2018, 10, 1161. [CrossRef] [PubMed]

5. Lova, P.; Manfredi, G.; Comoretto, D. Advances in functional solution processed planar 1D photonic crystals. Adv. Opt. Mater. 2018, 6, 1800730. [CrossRef]

6. Kang, H.S.; Lee, J.; Cho, S.M.; Park, T.H.; Kim, M.J.; Park, C.; Lee, S.W.; Kim, K.L.; Ryu, D.Y.; Huh, J. Printable and rewritable full block copolymer structural color. Adv. Mater. 2017, 29, 1700084. [CrossRef] [PubMed]

7. Lee, W.; Kim, S.; Kim, S.; Kim, J.-H.; Lee, H. Hierarchical opal grating films prepared by slide coating of colloidal dispersions in binary liquid media. J. Colloid Interface Sci. 2015, 440, 229-235. [CrossRef] [PubMed]

8. Kuo, W.-K.; Hsu J.-J.; Nien, C.-K.; Yu, H.H. Moth-eye inspired biophotonic surfaces with antireflective and hydrophobic characteristics. ACS Appl. Mater. Interfaces 2016, 8, 32021-32030 . [CrossRef] [PubMed] 
9. Nucara, L.; Piazza, V.; Greco, F.; Robbiano, V.; Cappello, V.; Gemmi, M.; Cacialli, F.; Mattoli, V. Ionic strength responsive sulfonated polystyrene opals. ACS Appl. Mater. Interfaces 2017, 95, 4818-4827. [CrossRef] [PubMed]

10. Nien, C.-K.; Yu, H.H. The applications of biomimetic cicada-wing structure on the organic light-emitting diodes. Mater. Chem. Phys. 2019, 227, 191-199. [CrossRef]

11. Zoorob, M.E.; Charlton, M.D.B.; Parker, G.J.; Baumberg, J.J.; Netti, M.C. Complete photonic bandgaps in 12-fold symmetric quasicrystals. Nature 2000, 404, 740-743. [CrossRef] [PubMed]

12. Zhang, X.; Zhang, Z.Q.; Chan, C.T. Absolute photonic bandgaps in 12-fold symmetric photonic quasicrystals. Phys. Rev. B 2001, 63, 081105R. [CrossRef]

13. Lai, N.D.; Lin, J.H.; Huang, Y.Y.; Hsu, C.C. Fabrication of two- and three-dimensional quasi-periodic structures with 12-fold symmetry by interference technique. Opt. Express 2006, 14, 10746-10752. [CrossRef] [PubMed]

14. Jia, L.; Bita, I.; Thomas, E.L. Level set photonic quasicrystals with phase parameters. Adv. Funct. Mater. 2012, 22, 1150-1157. [CrossRef]

15. Deubel, M.; von Freymann, G.; Wegener, M.; Pereira, S.; Busch, K.; Soukoulis, C.M. Direct laser writing of three-dimensional photonic-crystal templates for telecommunications. Nat. Mater. 2004, 3, 444-447. [CrossRef] [PubMed]

16. Nguyen, T.; Do, M.T.; Li, Q.; Tong, Q.C.; Au, T.H.; Lai, N.D. One-photon absorption-based direct laser writing of three-dimensional photonic crystals. In Theoretical Foundations and Application of Photonic Crystals; Vakhrushev, A., Ed.; INTech: Rijeka, Croatia, 2018; pp. 133-157. ISBN 978-953-51-3962-1.

17. Tong, Q.C.; Nguyen, D.T.T.; Do, M.T.; Luong, M.H.; Journet, B.; Ledoux-Rak, I.; Lai, N.D. Direct laser writing of polymeric nanostructures via optically induced local thermal effect. Appl. Phys. Lett. 2016, 108, 183104. [CrossRef]

18. Anderson, C.M.; Giapis, K.P. Larger two-dimensional photonic band gaps. Phys. Rev. Lett. 1996, 77, 2949. [CrossRef] [PubMed]

19. Horiuchi, N.; Segawa, Y.; Nozokido, T.; Mizuno, K.; Miyazaki, H. Isotropic photonic gaps in a circular photonic crystal. Opt. Lett. 2004, 29, 1084. [CrossRef] [PubMed]

20. Chang, D.; Scheuer, J.; Yariv, A. Optimization of circular photonic crystal cavities-Beyond coupled mode theory. Opt. Express 2005, 13, 9272. [CrossRef] [PubMed]

21. Do, M.T.; Nguyen, T.T.N.; Li, Q.; Benisty, H.; Ledoux-Rak, I.; Lai, N.D. Submicrometer 3d structures fabrication enabled by one-photon absorption direct laser writing. Opt. Express 2013, 21, 20964-20969. [CrossRef] [PubMed]

(C) 2019 by the authors. Licensee MDPI, Basel, Switzerland. This article is an open access article distributed under the terms and conditions of the Creative Commons Attribution (CC BY) license (http://creativecommons.org/licenses/by/4.0/). 